\section{Structure of the So-called cis Decalin}

THE structures of the two stereoisomeric hydrogenation products of naphthalene and their relationship to cyclohexane were discussed by one of us in $1943^{1}$. It was pointed out that the trans structure proposed by Mohr' ${ }^{2}$ (Fig. 1) will probably be correct, but reasons were given indicating that Mohr's cis structure containing two cyclohexane
rings of the 'cradle' form should be rejected in favour of another rings of the cradle' form should be rejected in favour of another molecules is found in the well-known diamond lattice. Using the notation introduced in a paper dealing with the cyclohexane problem ${ }^{3}$, notation introduced in a paper dealing with the cyclohexane problem ${ }^{3}$, $x$-bonds ; in cis-decalin, however, one $x$-bond and one $\varepsilon$-bond.

$x$-bonds ; in cis-decalin, however, one $x$-bond and one $\varepsilon$-bond. The correctness of these assumptions has been proved, using the electron diffraction technique and methods of computation worked out in this laboratory and based on the rotating sector method with subsequent Fourier analysis of the molecular scattering curves obtained from microphotometer records of the diffraction diagrams. The occurring when visual methods are used.

(a)

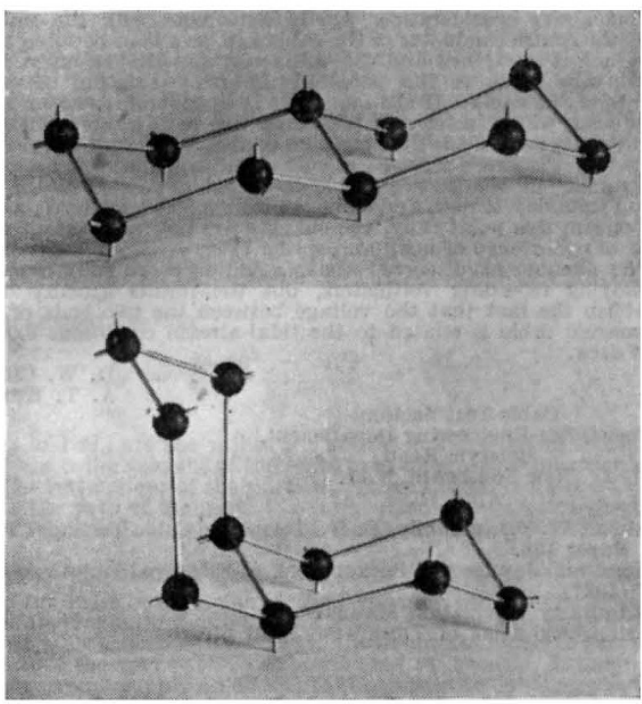

Fig. 1. STRUCTURE OF cis-DECALIN : (a) ACCORDING TO MOHR (b) ACCORDINg To BASTIANSEN AND HASSEL

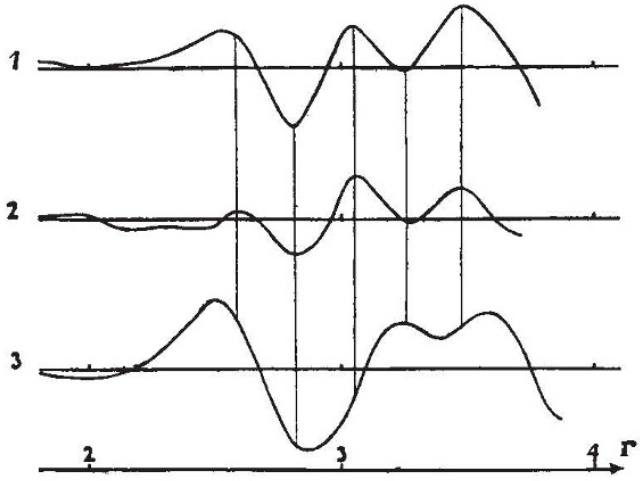

FIG. 2

The two $\sigma(r) / r$ curves giving the intramolecular distribution of nuclear distances in the two isomers show a high degree of conformity. We therefore preferred to discuss the differential curve obtained by subtracting the experimental $\sigma(r) / r$ curve of the trans form from that subtracting the experimental $\sigma(r) / r$ curve of the trans form from that
of the $c i s$ form. We then compared this curve with theoretical curves of the cis form. We then compared this curve with theoretical curves corresponding to our $x-\varepsilon$ model (Fig. $1 b$ ) of $c i s$-decalin and the structure
proposed by Mohr. The interval between $r=2$ and $r=4 \mathrm{~A}$. would proposed by Mohr. The interval to include the most pronounced differences in internuclear distances, and the three differential curves reproduced in Fig. 2 are : distances, and the three differential curves reproduced in Fig. 2 are : (1) theoretical curve for the cis $(-\varkappa \varepsilon)$ structure of Fig. $1 b$, (2) experimental
curve, and (3) theoretical curve for Mohr's eis structure, all related to curve, and (3) theoretical

A comparison of experimental and theoretical curves seems to settle beyond doubt that cis-decalin has the $x-\varepsilon$ structure given in Fig. $1 b$. The only symmetry element being a two-fold axis of symmetry, th cis-decalin should exist in two optically active forms.
A more detailed report will soon be published in Tidsskrift for Kjemi, Bergvesen og Metallurgi (Oslo).

O. Bastiansen

Chemical Institute

University, Oslo. A pril 13.

1 Hassel, O., Tid. Kjemi, Bergv., Met., 3, 91 (1943).

Mohr, E., Frdm. J. Chem., 98, 315 (1918).

Hassel, O., Tid. Kjemi, Bergv., Met., 3, 32 (1943).

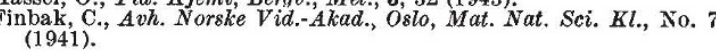

\section{Infra-Red Emissivity of Metals at High Temperatures}

A SPECIALLY designed vacuum-tight, water-cooled furnace constructed of silica and steel has been used to measure the emissivity of various metals at temperatures of $1,000^{\circ}-1,500^{\circ} \mathrm{C}$. and wave-lengths $1 \cdot 0-4 \cdot 5 \mu$.

The specimen, formed from a thin sheet bent into the form of a cylinder, was resistance-heated by a current of $300 \mathrm{amp}$. The intensity of the radiation from the inside and the outside of the cylinder was measured by means of a rock-salt prism spectrometer and a thermopile, and the ratio of these intensities after correction gave the emissivity. Thd the ratio of these intensities after correction gave the emissivity. The radiation from the leaving the two form, so for formed was not a could be compared. Results were obtained for pure iron and for platinum to an accuracy of about 2 per cent.

Sinum the an accuracy or about 2 per cent.

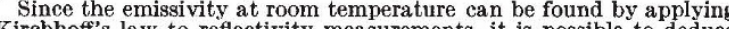
kirchoste to deduce the temperature coeffeient of emissivity in the near infra-red. This is of some importance, since it has been known for some time that transition regon must occur between the far infra-red region (where the Hagen-Rubens law predicts a coefficient equal to half the temperature coefficient of resistivity) and the visible region where the temperature variation is extremely small, and, in some cases, in the opposite direction to that predicted from electro-magnetic theory. A careful study of the literature has shown that in a number of instances result have been found indicating that, for a wave-length specific for each metal, the emissivity and reflectivity are constant over an extremely wide range of temperature. The results for tungsten given by the late Prof. Ornstein ${ }^{1}$ furnish the outstanding example of this constancy.

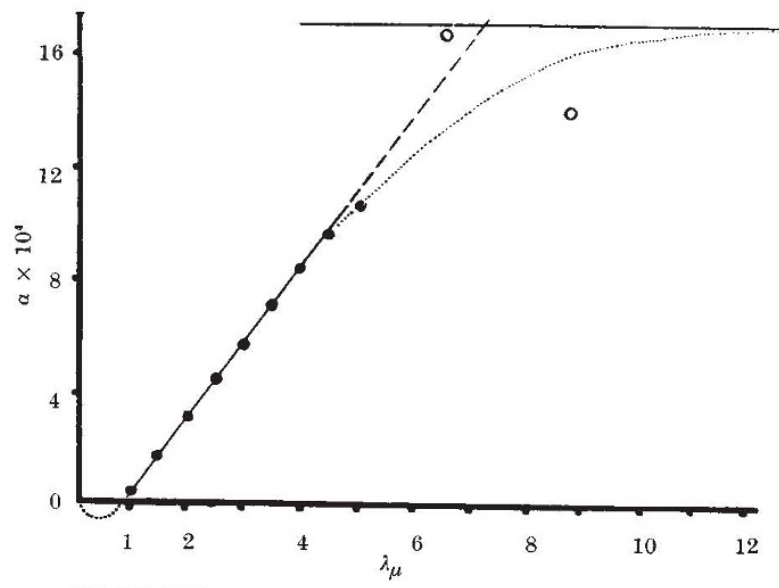

TEMPERATURE COEFFICIENT OF EMISSIVITY FOR PLATINUM

Horizontal line, Hagen-Rubens theoretical limit; 0 , HagenRubens experimental points ; $\bullet$, present experimental results.

The accompanying graph, based on the present work, shows the transition region for the temperature coefficient of emissivity of plat inum and indicates on extrapolation an $X$-point (wave-length of constancy) at about $0.93 \mu$. Results for pure iron give a similar straigh line showing a well-defined $X$-point at about $1 \cdot 0-1 \cdot 5 \mu$ (the uncertainty is due to ina ccuracies in the available data for room temperatur reflectivity). The origin of this $X$-point is as yet unknown. Work is at present in progress on molybdenum and nickel.

An interesting 'side-result' of these experiments has been the use of the determined efficiency curve of the cylinder biack-body to correct the hitherto unexplained deviation of Ives' Standard of Visible Radiation $^{2}$ from other independent determinations. A description of the apparatus together with more detailed experimental results wil be published elsewhere. A full summary of all previous work on the optical properties of metals with special reference to the problem of emissivity has also been made, and it is hoped that this will be published at a later date.

The above work forms part of a programme of research being undertaken for the Foundry Steel Temperature Sub-Committee of the Steel Castings Research Committee of the British Iron and Steel Research Association and the Iron and Steel Institute, and with their financia support.

S.W. Essex Technical College, D. J. Price

${ }^{1}$ Ornstein, Physica, 3, 561 (1917).

${ }^{2}$ Ives, J. Franklin Inst., 197 (1924) 